\title{
Effect of ambient temperature and attachment method on surface temperature measurements
}

\author{
Journal Article \\ Author(s): \\ Psikuta, Agnes; Niedermann, Reto; Rossi, René M. \\ Publication date: \\ 2014-07 \\ Permanent link: \\ https://doi.org/10.3929/ethz-b-000087577 \\ Rights / license: \\ In Copyright - Non-Commercial Use Permitted \\ Originally published in: \\ International Journal of Biometeorology 58(5), https://doi.org/10.1007/s00484-013-0669-4
}




\title{
Effect of ambient temperature and attachment method on surface temperature measurements
}

\author{
Agnes Psikuta • Reto Niedermann • René M. Rossi
}

Received: 26 September 2012 /Revised: 3 April 2013 /Accepted: 15 April 2013 /Published online: 19 May 2013

(C) ISB 2013

\begin{abstract}
Accurate measurement of skin surface temperature is essential in both thermo-physiological and clinical applications. However, a literature review of the last two decades of physiological or clinical research revealed an inconsistency or a lack of information on how temperature sensors were attached to the skin surface. The purpose of this study was to systematically compare and quantify the performance of different commercially available temperature sensors and their typical attachment methods, and, secondly, to provide a time-efficient and reliable method for testing any sensor-tape combination. In conclusion, both the sensor type and the attachment method influenced the results of temperature measurements (both its absolute and relative dimensions). The sensor shape and the contact of its sensing area to the surface, as well as the conductance of the tape were the most important parameters to minimise the influence of environmental conditions on surface temperature measurement. These results suggest that temperature sensors and attachment methods for human subject and manikin trials should be selected carefully, with a systematic evaluation of the sensor-tape system under conditions of use, and emphasise the need to report these parameters in publications.
\end{abstract}

Keywords Skin temperature measurement . Temperature sensor

\footnotetext{
A. Psikuta $(\triangle) \cdot$ R. Niedermann $\cdot$ R. M. Rossi

Laboratory for Protection and Physiology, Swiss Federal

Laboratories for Materials Science and Technology (EMPA),

Lerchenfeldstrasse 5,

9014 St. Gallen, Switzerland

e-mail: agnes.psikuta@empa.ch

R. Niedermann

Exercise Physiology, Institute of Human Movement

Sciences and Sport, ETH Zurich, Winterthurerstrasse 190,

8057 Zurich, Switzerland
}

\section{Introduction}

Accurate measurement of skin surface temperature is essential in both thermo-physiological and clinical applications (indication of hypovolemic shock, tissue injuries, vasomotor reactions, monitoring of post-operative patients, sleep research, non-invasive determination of core temperature, etc.). Introduction of a temperature sensor with an attachment tape to the surface always results in an unavoidable disturbance of the thermo-physical processes at the surface interface. However, a literature review of the last two decades of physiological or clinical research reported by Buono and Ulrich (1998) and Tyler (2011) revealed an inconsistency or a lack of information on how temperature sensors were attached to the skin surface. A similar trend was observed in manikin and clothing research for measurement of the fabric surface temperatures (Cheung and Sweeney 2001; Stoll and Hardy 1949).

Several studies have indicated that the measured surface temperature could depend on environmental conditions, the attachment method, and the type of sensor (Boetcher et al. 2009; Buono and Ulrich 1998; Harper Smith et al. 2010; Jirak et al. 1975). These studies were performed using either heated devices, such as thermal cylinder or manikin, or human subjects. The studies conducted on thermal devices involved mainly thermocouples (bare or fixed with various tapes) and some older sensors (Stoll and Hardy 1949; Cheung and Sweeney 2001). It was shown that bare or cemented thermocouples with a junction in direct contact with the surface provided the most accurate surface temperature measurements throughout various wind and radiation conditions. However, all these tests were done over a quite narrow range of temperature conditions $\left(\Delta \mathrm{T}_{\text {surface }- \text { ambient }}=\right.$ 10-19K).

Tests performed on human subjects at rest and exercise conditions included various commercially available sensors (Table 1). The reference temperature was measured either by 


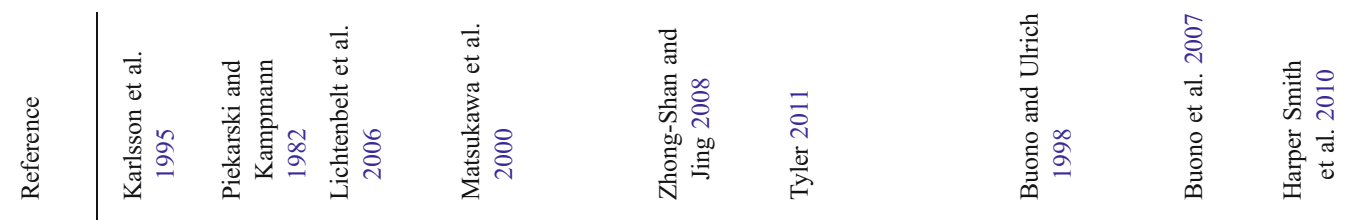

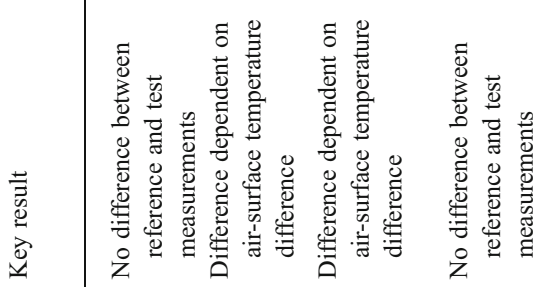

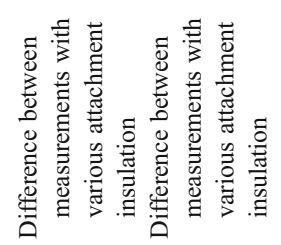

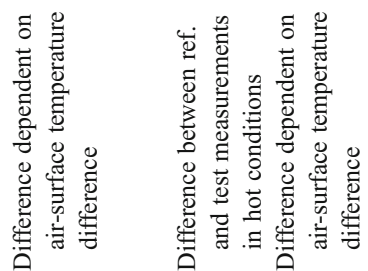

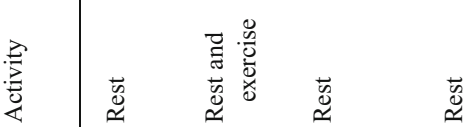

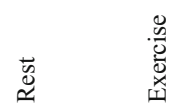

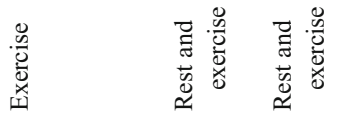

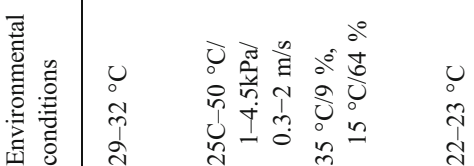

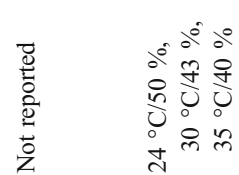

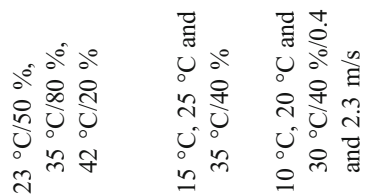

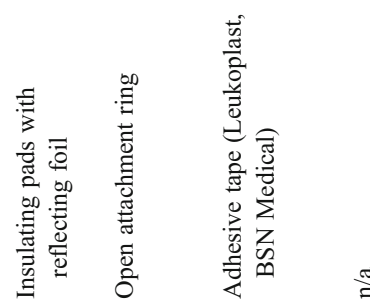

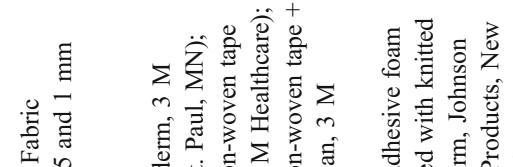
In

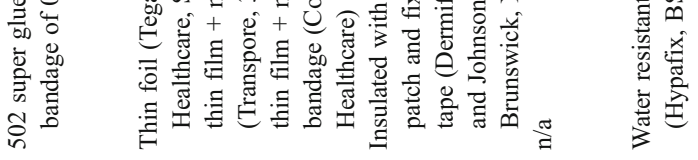

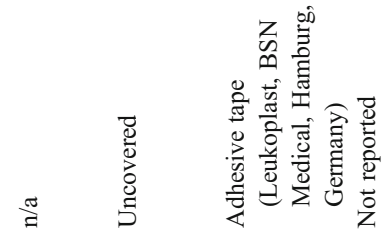

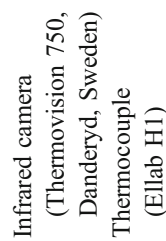
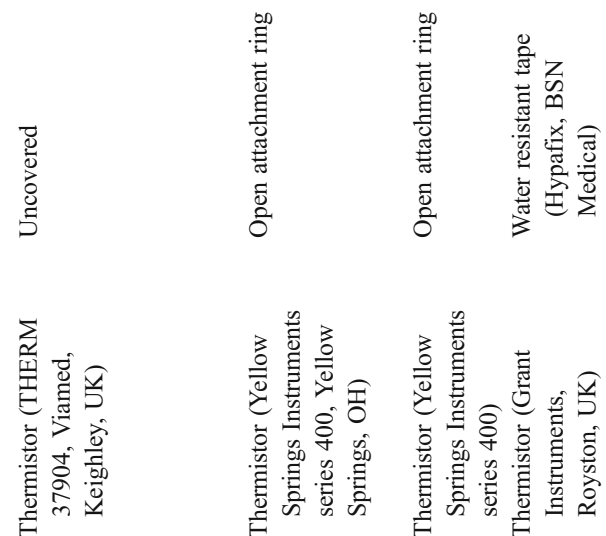

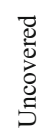

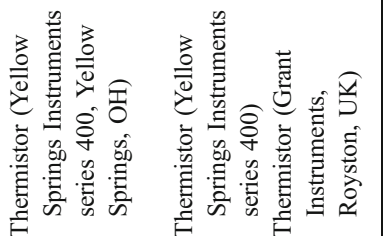

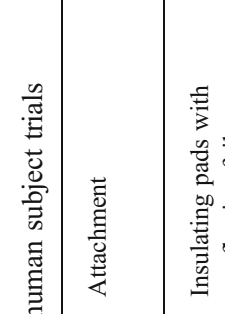


infrared camera, thermocouple or thermistor, which were used without any tape over the sensor in most cases. The results of these studies showed, in general, that both the temperature difference between the environment and the skin and the insulation added through the fixing tape affected the skin surface temperature measurement. However, they lacked any quantification of the discovered effects to allow appropriate correction of the results in subsequent measurements. They also lacked detailed information on the measurement conditions and reference temperature reliability. Furthermore, none of the studies provided detailed information on factors known to affect skin temperature measurement, for example, the pressure exerted by the sensors on the skin and underlying tissue. It is known that the sensor and its fixing method can influence skin blood perfusion, which may impose local artefacts on the temperature at the measurement point (Jirak et al. 1975; Mahanty and Roemer 1979; Stoll and Hardy 1950). Therefore, it is not possible to extract any correction factors for the influence of the temperature difference or fixing method as both observed effects include unknown artefacts of the pressure exerted on the skin. Similarly, infrared thermography was used in a few studies (Buono et al. 2007; Karlsson et al. 1995; Matsukawa et al. 2000) but the measurement protocol and calibration procedure were not reported. Recent studies reported on the need for a strict measurement protocol for good reproducibility (Zaproudina et al. 2008) and careful calibration for accurate absolute temperature measurement using infrared thermography (Grgic and Pusnik 2011).

In conclusion, no systematic study has been done that would:

(1) Collectively address all aspects such as environmental temperature, attachment method, and type of sensor for a selection of temperature sensors presently used in thermo-physiological measurements;

(2) Cover a large range of conditions allowing elaboration of a correction model for sensor-tape combinations;

(3) Recommend a low-labour-demanding method to determine the reliability of other sensors available on the market for use in physiological laboratories.

Secondly, it is difficult to derive correction factors based on the available human data due to incomplete information about measurement conditions as described above. A large database of strictly controlled human subject experiments would be necessary. On the other hand, thermal devices used in textile and clothing research are free of such constraints (no pressure and blood perfusion artefacts, integrated reference temperature sensors) and can provide comparable thermal conditions at the surface as the conditions on human skin (heat flow through convection, radiation and conduction from the vertical surface). The purpose of this study was to systematically compare and quantify the performance of different commercially available and frequently used temperature sensors attached to a heated plate using a selection of tapes and under various environmental conditions. Secondly, a relatively low-labour-demanding methodology to determine performance of sensor-tape combinations was developed in this study. Measurements were performed using a vertical heated plate that allowed the exclusion of pressure artefacts from the temperature measurements as well as providing repeatable surface conditions and ease of use. We also provide correction factors for the combinations of sensors and attachment methods used in this study.

\section{Methods}

Sensors and attachment methods

Four types of temperature sensors, namely, Pt100 foil sensors, MSR thermistors, Grant insulated temperature sensors and iButtons (Table 2), were placed in a horizontal row on the surface of the heated vertical plate and attached using one of four chosen tapes (Table 3). Both the sensors and the tapes were selected purposely to represent various configurations of sensor shapes and tape insulations commonly used in physiological and manikin studies.

\section{Sensor calibration}

All sensors used in this study were calibrated using an integrated humidity calibrator (Opti-Cal, Michell Instruments, Friedrichsdorf, Germany, calibrated by MCS, Altdorf, Switzerland to an accuracy of $\pm 0.1{ }^{\circ} \mathrm{C}$ ). The calibration protocol consisted of 1-h intervals of steady-state at air temperatures between $20{ }^{\circ} \mathrm{C}$ and $40{ }^{\circ} \mathrm{C}$ in steps of $5{ }^{\circ} \mathrm{C}$ while relative humidity was kept constant at $50 \%$ in the Opti-Cal. The data collected was used to obtain calibration coefficients for Pt100 sensors and to evaluate the performance of the sensors calibrated by the manufacturer (Grant sensors and iButtons). The Grant sensors and iButtons showed a temperature deviation lower than the accuracy given by the manufacturer $\left(-0.08\right.$ to $0.06{ }^{\circ} \mathrm{C}$ for Grant sensors with an accuracy of $\pm 0.3^{\circ} \mathrm{C}$ and -0.03 to $0.13^{\circ} \mathrm{C}$ for iButtons with an accuracy of $\left.\pm 0.5^{\circ} \mathrm{C}\right)$.

\section{Heated plate}

The heated plate used in this study consisted of an aluminium slab $66 \mathrm{~cm} \times 54 \mathrm{~cm}$ large and $1 \mathrm{~cm}$ thick, which was heated with strip heating elements attached to the back surface and insulated with a 4-cm thick mineral wool block. The temperature of the plate was controlled within $\pm 0.03{ }^{\circ} \mathrm{C}$ 
Table 2 Sensor specifications

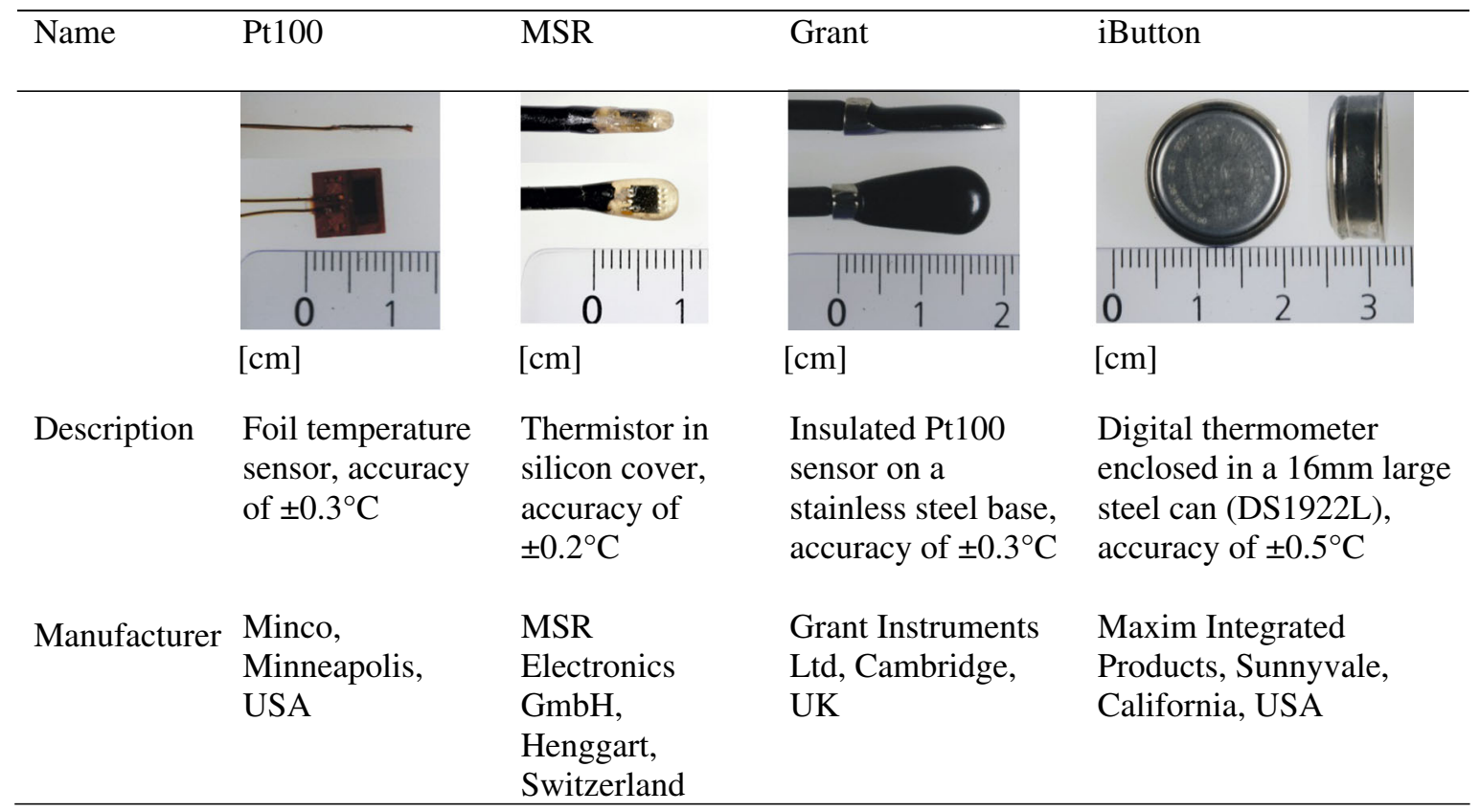

by a dedicated PID controller adjusting power supply based on readings of the Pt100 foil temperature sensor (Minco, Minneapolis, MN) embedded in the middle and within the aluminium slab. The outer surface of the plate was painted flat black to resemble the emissivity of human skin of 0.98 (Steketee 1973). The central part $(30 \mathrm{~cm} \times 20 \mathrm{~cm})$ was additionally monitored with an IR camera (Flir A40, Flir Systems, http://www.flir.com, sensitivity of $<0.1{ }^{\circ} \mathrm{C}$ ) to confirm the surface temperature homogeneity (observed differences of less than $0.1^{\circ} \mathrm{C}$ ) in the area where the sensors were attached.

\section{Measurement protocol}

The sensors were placed on the surface of the heated vertical plate and attached by the respective tape. The temperature of the heated plate was maintained constant at $36.5^{\circ} \mathrm{C}$. After an initial stabilisation period, surface temperature measurements were taken using the attached sensors. The ambient air temperature was subsequently set at $15^{\circ} \mathrm{C}, 20^{\circ} \mathrm{C}, 25^{\circ} \mathrm{C}, 30^{\circ} \mathrm{C}$ and $35^{\circ} \mathrm{C}$, the relative humidity at $50 \%$, and the air velocity at $0.5 \mathrm{~m} / \mathrm{s}$ (monitored using thermal conditions monitoring system, ThermCondSys5500, Sensor Electronic, Poland) . Measurements at each ambient air temperature were repeated three times. All sensors were detached and attached again to the surface of the heated plate for each repetition in order to evaluate the repeatability of the attachment process. In the second part of the experiment, a set of fans was used to produce an air movement of $1.2 \mathrm{~m} / \mathrm{s}$ (corresponding to walking speed) in a predominantly horizontal and parallel direction to the surface of the heated plate at ambient air temperatures of $15^{\circ} \mathrm{C}, 25^{\circ} \mathrm{C}$ and $35^{\circ} \mathrm{C}$.

Data analysis

To quantify the change of the surface temperature sensor readings in relation to the change of ambient air temperature, the sensor type, and the attachment method, the correction coefficients (corr, ${ }^{\circ} \mathrm{C} /{ }^{\circ} \mathrm{C}$ of temperature difference between surface and ambient air) were determined. We used regression analysis to correlate temperature difference between the surface and the environment and the deviation of the measured and actual surface temperatures for each attachment-sensor combination. The correlation could be described by linear approximation in the investigated environmental temperature range and the correlation coefficient could be defined as:

corr $=\frac{\left(\mathrm{T}_{\text {surf,real }}-\mathrm{T}_{\text {surf,meas }}\right)}{\left(\mathrm{T}_{\text {surf,real }}-\mathrm{T}_{\text {ambient }}\right)}$

which, after rearranging the corrected surface temperature formulae, gives:

$\mathrm{T}_{\text {surf,real }}=\frac{\left(\mathrm{T}_{\text {surf,meas }}-\text { corr } \cdot \mathrm{T}_{\text {ambient }}\right)}{1-\text { corr }}$,

where $\mathrm{T}_{\text {surf,real }}$ is the actual surface temperature $\left({ }^{\circ} \mathrm{C}\right)$, $\mathrm{T}_{\text {surf,meas }}$ is the measured surface temperature using the given sensor and attachment method $\left({ }^{\circ} \mathrm{C}\right)$, and $\mathrm{T}_{\text {ambient }}$ is the ambient air temperature $\left({ }^{\circ} \mathrm{C}\right)$. 
Table 3 Attachment tapes

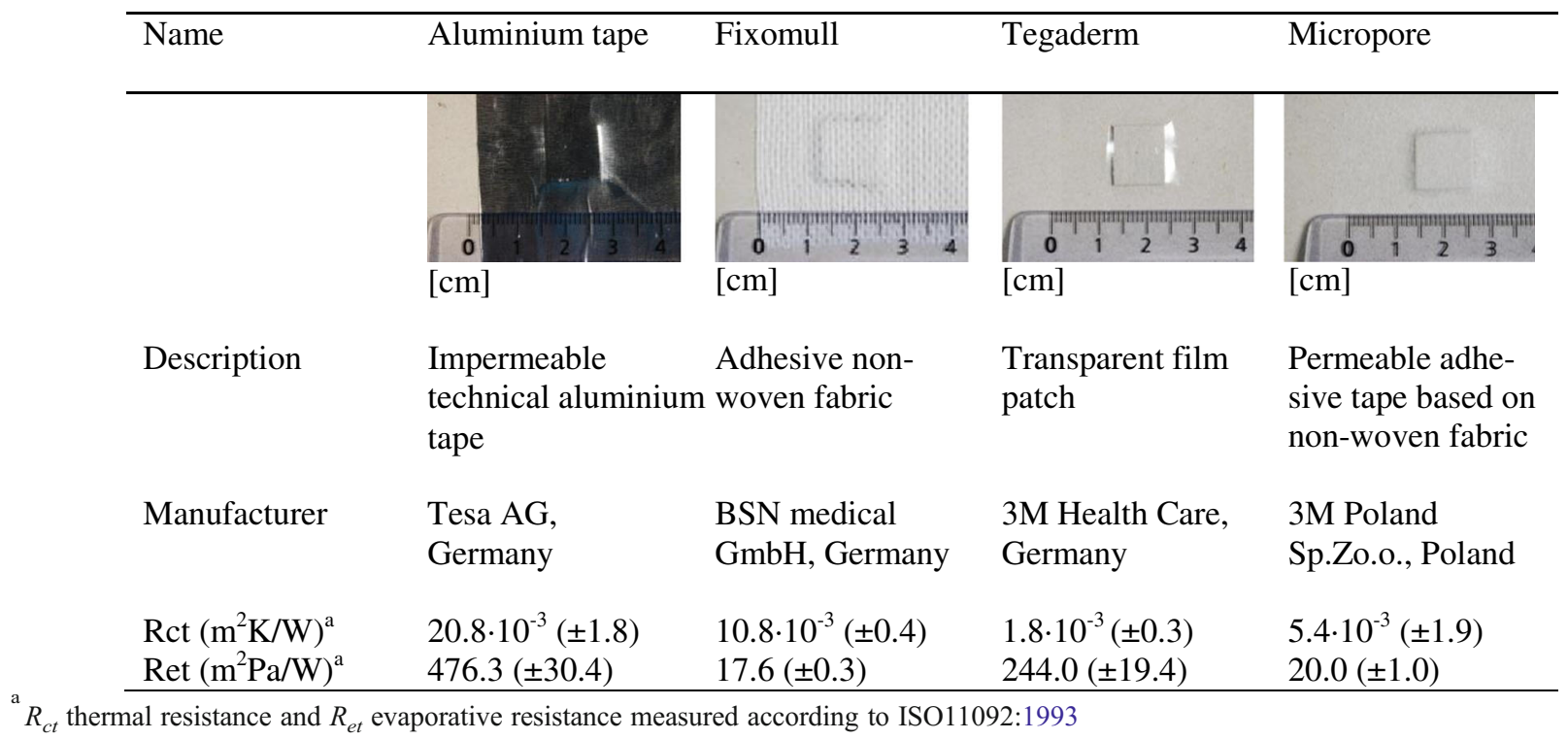

\section{Results}

Figure 1 shows the surface temperature of the heated plate measured using all combinations of four sensors and four fixing tapes. Table 4 lists the derived correction coefficients (Slope), their standard errors ( $\left.\mathrm{SE}_{\text {slope }}\right)$ and coefficients of determination $\left(R^{2}\right)$. Figure 2 shows the change in surface temperature measurement in relation to the ambient temper- ature in conditions with low $(0.5 \mathrm{~m} / \mathrm{s})$ and high $(1.2 \mathrm{~m} / \mathrm{s})$ air velocity.

\section{Discussion}

Well-defined linear trends in surface temperature measurement in relation to the ambient temperature were observed
Fig. 1 Surface temperatures of the heated plate measured using Pt100 foil sensors, MSR thermistors, Grant insulated temperature sensors and iButtons attached using respective tapes, and under different ambient temperatures
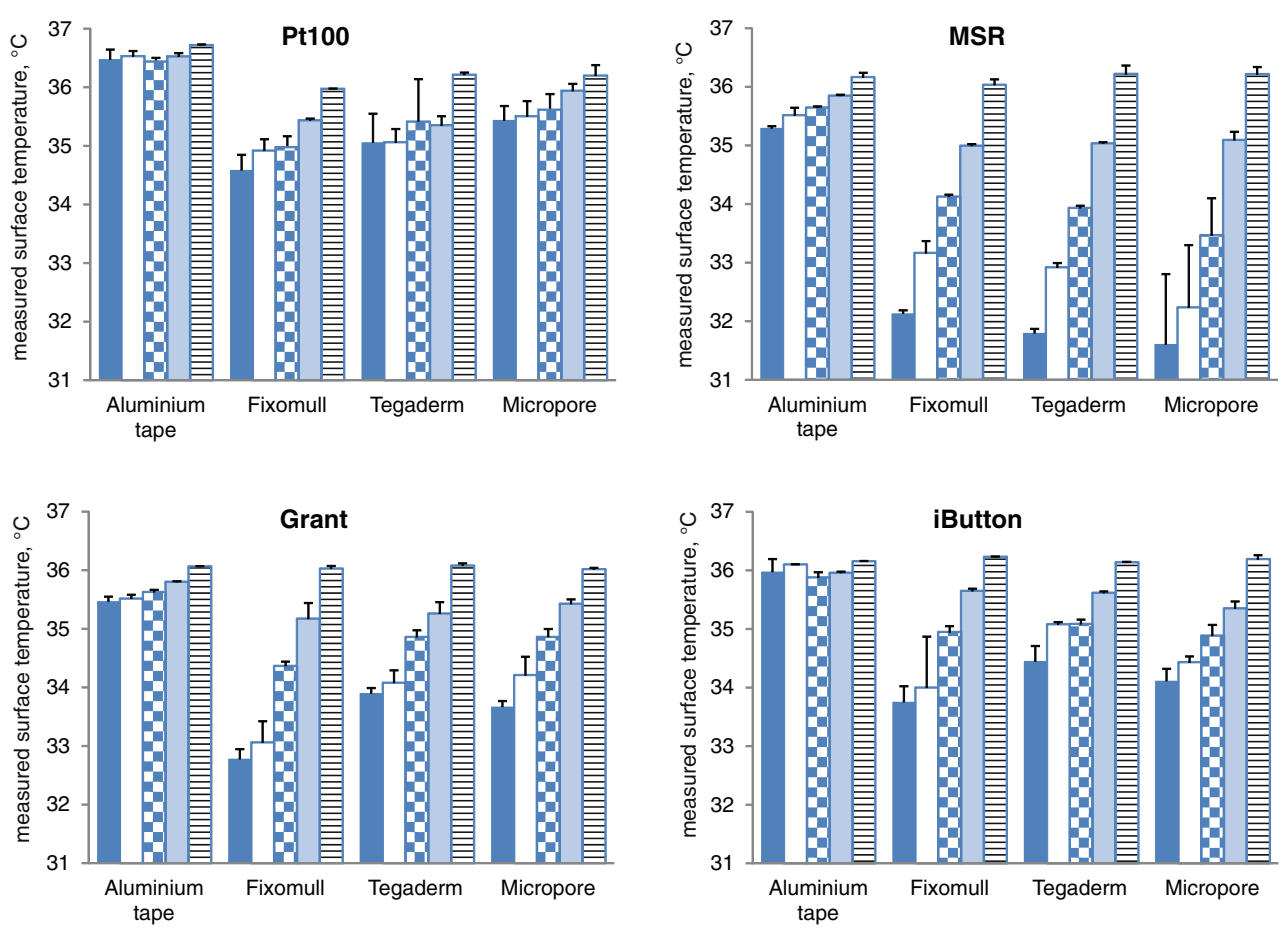

Grant
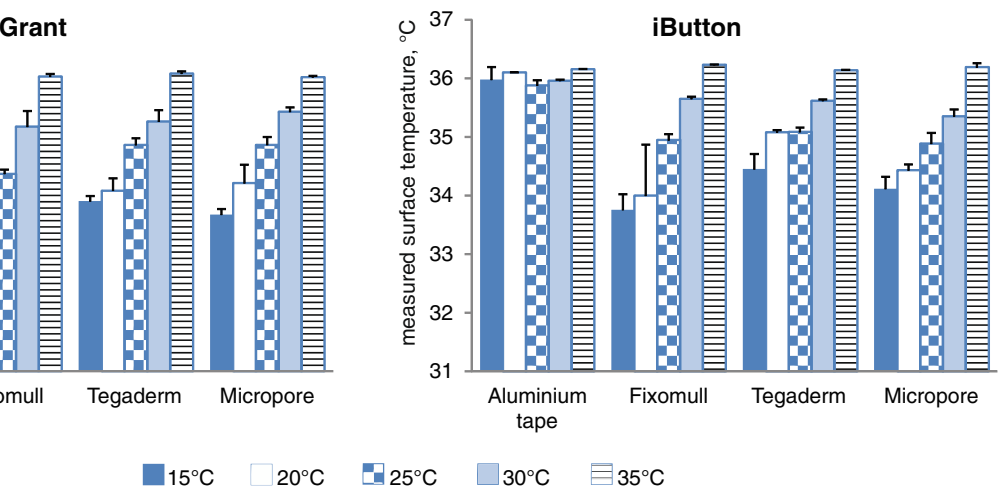
Table 4 Change in surface temperature sensor readings in ${ }^{\circ} \mathrm{C} /{ }^{\circ} \mathrm{C}$ in relation to the temperature difference between plate surface and ambient air at low air velocity (slope is a change of sensor readings in relation to temperature difference in ${ }^{\circ} \mathrm{C} /{ }^{\circ} \mathrm{C}$, $\mathrm{SE}_{\text {slope }}$ is the standard error of the slope and $R^{2}$ is the coefficient of determination)

\begin{tabular}{llllll}
\hline \multicolumn{2}{c}{ Attachment method } & Aluminium tape & Fixomull & Tegaderm & Micropore \\
\hline \multirow{2}{*}{ Pt100 } & Slope & 0.003 & 0.110 & 0.090 & 0.065 \\
& SE $_{\text {slope }}$ & 0.004 & 0.014 & 0.014 & 0.008 \\
\multirow{3}{*}{ MSR } & $R^{2}$ & 0.14 & 0.94 & 0.91 & 0.94 \\
& Slope & 0.068 & 0.220 & 0.235 & 0.258 \\
& SE & 0.008 & 0.006 & 0.002 & 0.009 \\
\multirow{3}{*}{ slope } & $R^{2}$ & 0.95 & 1.00 & 1.00 & 1.00 \\
& Slope & 0.065 & 0.201 & 0.146 & 0.148 \\
& SE & 0.011 & 0.010 & 0.011 & 0.008 \\
\multirow{3}{*}{ iButton } & $R^{2}$ & 0.90 & 0.99 & 0.98 & 0.99 \\
& Slope & 0.036 & 0.147 & 0.107 & 0.133 \\
& SE $_{\text {slope }}$ & 0.011 & 0.006 & 0.009 & 0.010 \\
& $R^{2}$ & 0.74 & 0.99 & 0.97 & 0.98 \\
\hline
\end{tabular}

(Fig. 1) for the investigated environmental temperature range. A relatively large variation in the measured surface temperatures was observed for all sensors fixed with Fixomull, Tegaderm and Micropore. The influence of environmental conditions on surface temperature measurements was clearly dependent on the sensor shape and the contact of its sensing area to the surface. The Pt100 foil sensor was the flattest and had a large contact area to the surface, which resulted in the most stable performance under all environmental conditions. Conversely, the most rounded MSR sensor showed the largest dependence on ambient temperature changes.

For all sensors tested, the smallest variation was found for the aluminium tape. This fact most probably resulted from the good thermal conductance of the aluminium tape. This means that the heat from the heated plate was also

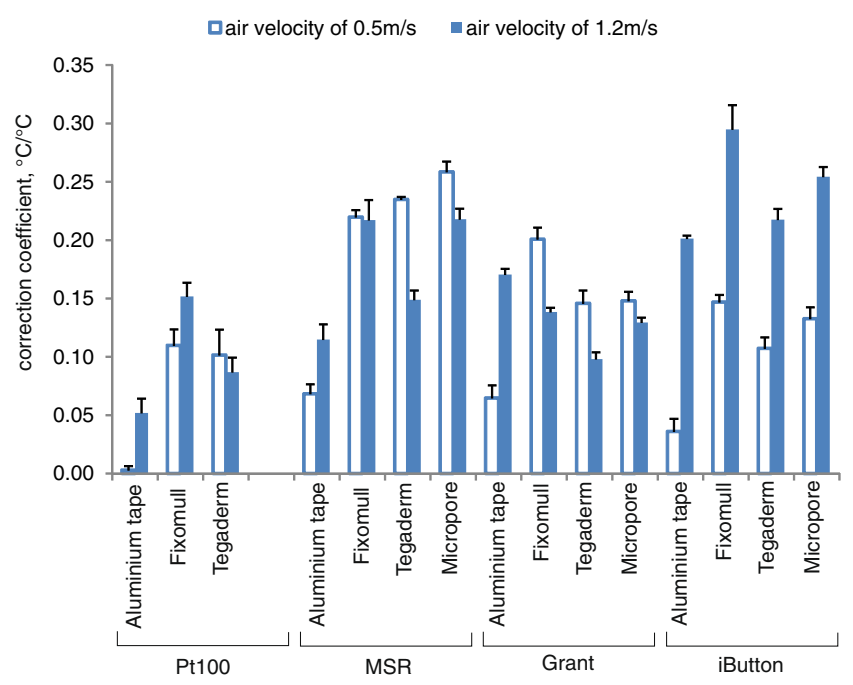

Fig. 2 Change of surface temperature measurement on the heated plate due to change of ambient temperature in conditions with low $(0.5 \mathrm{~m} / \mathrm{s})$ and higher $(1.2 \mathrm{~m} / \mathrm{s})$ air velocity conducted over the sensor, and thus, it reduced the influence of the ambient temperature on the sensor beneath the tape. This fact is in accordance with the study by Boetcher et al. (2009), who recommended a highly conductive cover of surface temperature sensor. A similar effect was visible in the measurements performed using iButtons, where the sensor itself is encapsulated in a heat-conducting stainless steel can. Effectively, in general, iButtons exhibited rather small changes in surface temperature readings despite their bulky size.

No clear influence of the thermal resistance of the fixing tape was observed in this study. Excluding the aluminium tape with its special conductive feature, the largest thermal insulation was represented by Fixomull tape. However, its performance was comparable with the other less-isolative (2-5 times) tapes. Therefore, the effect of shape of the sensor and its surface contact seems to surpass the effect of the thermal resistance of the tape in this study. On the other hand, when multiple layers of tape or foam patches were used (Tyler 2011; Boetcher et al. 2009; Buono and Ulrich 1998; Zhong-Shan and Jing 2008), a significant overestimation of the temperature measurement occurred. However, the thermal insulation of the attachment tape in those studies was higher (thermal resistance $>0.02 \mathrm{~m}^{2} \mathrm{~K} / \mathrm{W}$ ) than that of the selection of tapes used in this study (thermal resistance $<0.01 \mathrm{~m}^{2} \mathrm{~K} / \mathrm{W}$ ).

The correction coefficients obtained with our experiments were used to correct skin temperatures in resting subjects measured in the study of Harper Smith et al. (2010) (Fig. 3). This direct comparison was possible as the sensors (Grant thermistors and iButtons) and the Hypafix tape (BSN Medical, Hamburg, Germany - similar to Fixomull produced by the same company) used in the study by Harper Smith et al. (2010) were the same or comparable to those used in our study. After the correction of the data of Harper Smith et al. (2010) for ambient-to-skin temperature difference, the average 
Fig. 3 Comparison between mean skin temperatures in resting subjects measured using iButtons and Grant thermistors attached using the Hypafix tape (open symbols, data of Harper

Smith et al. 2010) and corrected using respective correction coefficients from Table 4 (iButtons and Grant thermistors combined with Fixomull tape, solid symbols). $T_{a}$ Ambient temperature; $r m s d_{\text {orig }}$, rmsd $_{\text {corr }}$ root-mean-square deviations; $\Delta T_{\text {orig }}, \Delta T_{\text {corr }}$ skin temperature differences between the beginning and the end of the exposure for original and corrected data sets, respectively

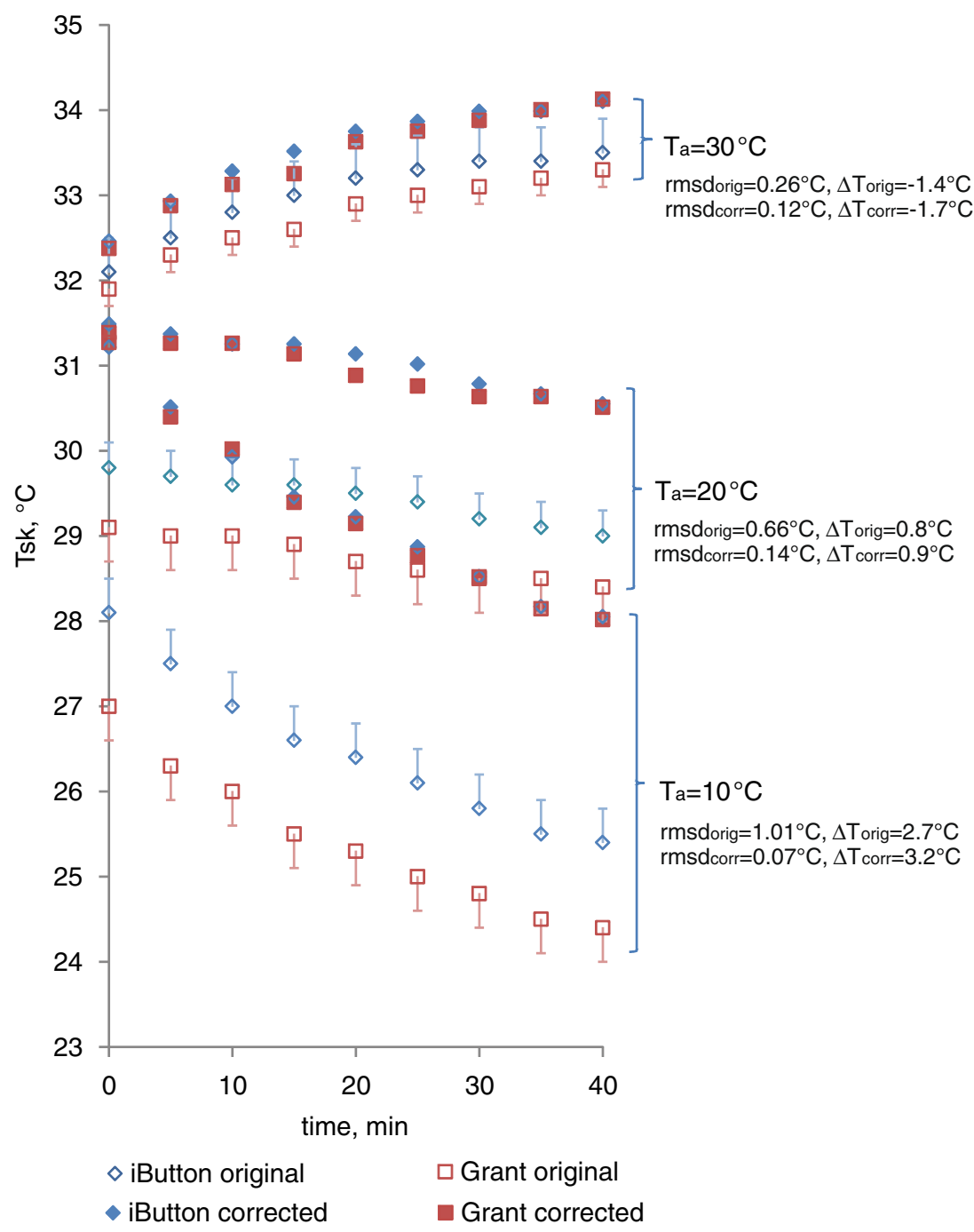

difference between measurements using both types of sensors (rmsd in Fig. 3) decreased, and fell within the accuracy range guaranteed by the sensor's manufacturers (Table 2). This suggests that correction coefficients developed based on measurements made on the vertical flat plate would be also applicable for correction of skin temperature in human subjects in relatively dry conditions (resting subjects, possibly only in the presence of undetectable perspiration).

Secondly, not only absolute temperatures but also relative differences in skin temperatures were affected by the ambientto-skin temperature difference corrections. This is an important issue in studies where temperature difference is measured (e.g. effect of a treatment), and it is assumed that possible measurement offsets would cancel out if both temperatures were measured by the same sensor-tape combination. As the temperature correction is proportional to the ambient-to-skin temperature difference, it will change if either skin or ambient temperature change. For example, the skin temperature change between the beginning and the end of the exposure period ( $\Delta \mathrm{T}$ in Fig. 3 ) has changed due to different magnitudes of the ambient-to-skin temperature differences at the beginning and the end of the exposure. Similar issues may occur when considering differences between body parts at different temperatures (e.g. hand-forearm for indication of vasomotor response) or temperatures of nude and covered skin (e.g. mean skin temperature of a heterogeneously dressed person).

Under windy conditions $(1.2 \mathrm{~m} / \mathrm{s})$, the linear trends in surface temperature measurement in relation to the ambient temperature were maintained, and the correction coefficient could be derived based on regression analysis and Eq. 1. It was expected that these correction coefficients will be larger than those for low air velocity $(0.5 \mathrm{~m} / \mathrm{s})$ due to increased heat transfer in the turbulent air layer at the surface with attached sensors. However, this trend was observed only for iButtons and for all sensors fixed with the aluminium tape (Fig. 2). Grant and MSR sensors in combination with the non-metallic tapes showed less pronounced and typically reverse trends. It seems that the correction coefficients of the sensors covered by thermally conducting material 
(aluminium tape, steel can of iButtons) were more prone to changes of air movement conditions at the surface (average correction coefficient increase of $0.12{ }^{\circ} \mathrm{C} /{ }^{\circ} \mathrm{C}$ in a range of 0.05-0.17) than other sensor-tape combinations (average correction coefficient increase of $-0.03{ }^{\circ} \mathrm{C} /{ }^{\circ} \mathrm{C}$ in a range of $-0.09-0.04)$. This comparison showed the complexity of the surface temperature measurement at various environmental conditions using different sensor-tape combinations and revealed the necessity of systematic evaluation of the sensor-tape system under conditions of future use in physiological and manikin studies.

In the studies where covering sensors with any fabric is required (measurements on thermal devices or human subject trials), the additional fabric layer will reduce the temperature difference between the surface and the air in the proximity of the sensor. This scenario is, however, already addressed in the correction coefficients as they were developed for a range of temperature differences between 1.5 and $21.5 \mathrm{~K}$. Secondly, the air gap between the fabric and the surface such as human skin can vary significantly depending on the body part, type of the garment design, etc. affecting the temperature inside the air gap (Wissler and Havenith 2009, Psikuta et al. 2012). The air gap shape and its influence on the thermal processes in the clothing is a very complex issue, and thus, it would be easier to measure its resultant effect directly on the human subject by measuring the clothing microenvironment temperature. Therefore, to use the correction coefficients for dressed subjects, the additional air temperature in the clothing microenvironment should be taken or alternatively an estimation of this temperature could be done using, for example, ISO9920 (2007).

Human skin is in general softer than the plate surface, which may lead to the increased contact area between the sensor and the skin as compared to the hard plate. This issue is particularly valid for sensors of round shape such as MSR (see Table 2). The other sensors have a flat sensing area and positioning them on the softer surface may not have any noticeable effect on the surface temperature measurement. This is actually a desired feature of the temperature sensors used in physiological studies in order to remain independent from artefacts of various levels of skin softness (skin directly on the bone such as at shin or forehead, skin on tensed or relaxed muscle, various fat contents of human subjects).

There is a variety of applications for the skin temperature measurement as well as the surface temperature measurement of thermal devices and textile layers. The necessary accuracy of surface temperature measurement is dependent on the actual research questions and experiment design and only the researcher can take the decision on whether to correct the measurements. There are some situations where it is recommended to consider correcting the temperature measurements, namely:
- Large temperature differences between the surface and ambient air in the proximity of the sensor;

- Transient conditions during exposure, i.e. temperature difference between the surface and ambient air has changed significantly during exposure and some comparisons before/after are required;

- Heterogeneous conditions at various body parts (human subject or thermal manikin), for example, uncovered areas (e.g. head and hands) under cool conditions, which offer significantly lower insulation on the lower body than on the upper body.

\section{Conclusion}

This study shows clearly that both the sensor type and the attachment method influence the results of temperature measurements in both absolute and relative dimensions. The sensor shape and the contact of its sensing area to the surface, as well as the conductance of the tape, are the most important parameters to consider to minimise the influence of environmental conditions on surface temperature measurements. These results suggest that temperature sensors and attachment methods for surface measurements be selected carefully in both human subject and thermal devices or manikin trials. They also address the need to performing a systematic evaluation of the sensor-tape system under the actual conditions of use. The performance assessment method developed in this study was shown to be applicable to correcting skin temperature measurements in resting subjects. Finally, this investigation emphasises the need to report testing details and correction parameters in publications.

Acknowledgements Part of this study was supported financially by EU project Prospie (FP7-NMP-229042). The authors thank the members of the Prospie consortium for making available some sensor systems and for their constructive feedback, and Dr. Veronika Meyer from Laboratory for Protection and Physiology at Empa for her editorial input.

\section{References}

Boetcher SKS, Sparrow EM, Dugay MV (2009) Characteristics of direct-contact, skin-surface temperature sensors. Int J Heat Mass Transf 52(15-16):3799-3804

Buono MJ, Ulrich RL (1998) Comparison of mean skin temperature using 'covered' versus 'uncovered' contact thermistors. Physiol Meas 19(2):297-300

Buono MJ, Jechort A, Marques R, Smith C, Welch J (2007) Comparison of infrared versus contact thermometry for measuring skin temperature during exercise in the heat. Physiol Meas 28(8):855-859 
Cheung SS, Seweeney DH (2001) The influence of attachment method and clothing on skin temperature sensor accuracy. Med Sci Sports Exerc 33(5): 161

Deng Z, Liu J (2008) Effect of fixing material on skin-contact temperature measurement by wearable sensor. 2008 5th International Summer School and Symposium on Medical Devices and Biosensors, 1-3 June 2008, Hong Kong, China, pp 137-140

Grgic G, Pusnik I (2011) Analysis of thermal imagers. Int J Thermophys 32(1-2):237-247

Harper Smith AD, Crabtree DR, Bilzon JLJ, Walsh NP (2010) The validity of wireless iButtons $(\mathrm{R})$ and thermistors for human skin temperature measurement. Physiol Meas 31 (1):95-114

ISO9920 (2007) Ergonomics of the thermal environment - estimation of thermal insulation and water vapour resistance of a clothing ensemble

ISO11092 (1993) Textiles - physiological effects - measurement of thermal and water-vapour resistance under steady-state conditions (sweating guarded-hotplate test)

Jirak Z, Jokl M, Stverak J, Pechlat R, Coufalova H (1975) Correction factors in skin temperature measurement. J Appl Physiol 38(4):752-756

Karlsson H, Hanel SE, Nilsson K (1995) Evaluation of methods for measurement of regional skin temperature and heat flow in neonates. Acta Paediatr 84(6):599-603

Lichtenbelt WDV, Daanen HAM, Wouters L, Fronczek R, Raymann R, Severens NMW, Van Someren EJW (2006) Evaluation of wireless determination of skin temperature using iButtons. Physiol Behav 88(4-5):489-497
Mahanty DD, Roemer RB (1979) The effect of pressure on skin temperature measurements for a disk sensor. Trans ASME, J Biomech Eng 101(4):261-266

Matsukawa T, Ozaki M, Nishiyama T, Imamura M, Kumazawa T (2000) Comparison of infrared thermometer with thermocouple for monitoring skin temperature. Crit Care Med 28(2):532-536

Piekarski C, Kampmann B (1982) Zur Messung der Hauttemperatur mit Thermistorfühlern. Arbeitsmedizin Sozialmedizin Präventivmedizin 17(11):268-271

Psikuta A, Frackiewicz-Kaczmarek J, Frydrych I, Rossi RM (2012) Quantitative evaluation of air gap thickness and contact area between body and garment. Text Res J 82(14):1405-1413

Steketee J (1973) Spectral emissivity of skin and pericaredium. Phys Med Biol 18(5):686-694

Stoll AM, Hardy JD (1949) Direct experimental comparison of several surface temperature measuring devices. Rev Sci Instrum 20(9):678-686

Stoll AM, Hardy JD (1950) Study of thermocouples as skin thermometers. J Appl Physiol 2(10):531-543

Tyler CJ (2011) The effect of skin thermistor fixation method on weighted mean skin temperature. Physiol Meas 32(10):1541-1547

Wissler EH, Havenith G (2009) A simple theoretical model of heat and moisture transport in multi-layer garments in cool ambient air. Eur J Appl Physiol 105(5):797-808

Zaproudina N, Varmavuo V, Airaksinen O, Narhi M (2008) Reproducibility of infrared thermography measurements in healthy individuals. Physiol Meas 29(4):515-524 